\title{
BROTE DE SÍNDROME TÓXICO DEL SEGMENTO ANTERIOR DESPUÉS DE CIRUGÍA VÍTREA
}

\section{OUTBREAK OF TOXIC ANTERIOR SEGMENT SYNDROME AFTER VITREOUS SURGERY}

\author{
ANDONEGUI J ${ }^{1}$, JIMÉNEZ-LASANTA L ${ }^{1}$, ALISEDA D ${ }^{2}$, LAMEIRO F ${ }^{1}$
}

\begin{abstract}
RESUMEN
Caso clínico: Se describe un brote de Síndrome Tóxico del Segmento Anterior después de cirugía vítrea. Dos pacientes habían sido intervenidos exclusivamente mediante vitrectomía mientras que los otros tres fueron sometidos a vitrectomía y algún otro procedimiento del segmento anterior.

Discusión: El Síndrome Tóxico del Segmento Anterior es una inflamación postoperatoria estéril provocada por alguna sustancia no infecciosa que alcanza el polo anterior durante la cirugía. Suele ocurrir en brotes y aunque la mayoría de los casos han sido descritos después de cirugía del segmento anterior, los casos que presentamos demuestran que es posible su aparición tras cirugía vítrea.
\end{abstract}

Palabras clave: Síndrome tóxico segmento anterior, vitrectomía.

\begin{abstract}
Case report: An outbreak of Toxic Anterior Segment Syndrome after vitreoretinal surgery is reported. Two patients underwent exclusively vitrectomy while the other three patients were operated of vitrectomy and some other anterior segment procedure.

Discussion: Toxic Anterior Segment Syndrome is a sterile postoperative inflammation due to any non infectious substance that reaches the anterior segment during surgery. It occurs in outbreaks and while most of the cases have been reported after anterior segment procedures, this case demonstrates that development after vitreoretinal surgery is also a possibility (Arch Soc Esp Oftalmol 2009; 84: 403-406).
\end{abstract}

Key words: Toxic anterior segment syndrome, vitrectomy.

cirugía y provoca un daño tóxico en los tejidos intraoculares $(1,2)$. Suele ocurrir en brotes y la mayoría de los casos han sido descritos después de cirugía del segmento anterior. Describimos un brote de STSA aparecido después de cirugía vítreoretiniana.

\footnotetext{
Recibido: 31/5/07. Aceptado: 4/8/09.

Servicio de Oftalmología. Hospital de Navarra. 31008 Pamplona (España).

1 Licenciado en medicina. Hospital de Navarra. Pamplona. Navarra. España.

2 Doctor en medicina. Hospital de Navarra. Pamplona. Navarra. España.

Correspondencia:

José Andonegui Navarro

Servicio de Oftalmología

Hospital de Navarra

31008 Pamplona

España

E-mail: jandonen@cfnavarra.es
} 


\section{CASOS CLÍNICOS}

Se trata de cinco pacientes sometidos a cirugía vítrea asociada o no a algún procedimiento del segmento anterior e intervenidos en dos sesiones quirúrgicas consecutivas realizadas en el mismo quirófano con cuatro días de separación. En la primera sesión fueron intervenidos tres pacientes, de los cuales dos desarrollaron el síndrome, mientras que en la segunda sesión este apareció en los tres pacientes operados. En la primera sesión fue intervenido mediante vitrectomía otro paciente que presentaba una membrana epirretiniana y no resultó implicado, aunque en esta cirugía se empleó el mismo tipo de solución salina balanceada (BSS) y los mismos sistemas de limpieza y esterilización. Los cinco casos afectados presentaban a las 24 horas de la cirugía una reacción inflamatoria en cámara anterior caracterizada por intensa turbidez del humor acuoso, redes de fibrina e hipopion. Los dos primeros fueron diagnosticados erróneamente de endoftalmitis infecciosa y reintervenidos mediante vitrectomía, toma de cultivo y antibióticos intravítreos. En ambos el cultivo resulto estéril, lo que oriento el diagnóstico hacia el STSA y se estableció tratamiento mediante corticoides tópicos de forma frecuente.
Los otros tres casos fueron tratados inicialmente mediante corticoides tópicos. En los cinco pacientes la reacción inflamatoria desapareció gradualmente entre 4 y 7 días después de la intervención. Uno de los casos intervenidos mediante vitrectomía desarrolló un desprendimiento de retina operado con buen resultado mediante cerclaje, vitrectomía e intercambio con gas. Las características clínicas de estos pacientes aparecen expuestas en la tabla I.

Una vez establecido el diagnóstico de STSA, se llevaron a cabo varias medidas para erradicar el brote. En primer lugar, se sustituyó el BSS empleado, que se elaboraba en la farmacia del hospital, por otro de fabricación comercial. Se realizó un análisis microbiológico del BSS, que resultó negativo. Se analizaron los niveles de endotoxinas tanto del BSS como del agua del grifo empleada para limpiar el instrumental tras la cirugía, estando en ambos casos en límites considerados aceptables. Se comenzó a utilizar agua destilada estéril en vez de agua del grifo para el baño de ultrasonidos. Por último, todos los lotes de sustancias que pudieran ser introducidas en el interior del ojo durante la cirugía (adrenalina, viscoelásticos, cefuroxima), fueron sustituidos por otros nuevos. En las siguientes sesiones quirúrgicas no se detectaron nuevos casos.

Tabla I. Características clínicas e intraoperatorias de los pacientes

\begin{tabular}{|c|c|c|c|c|c|c|c|c|}
\hline Paciente & Edad & Sexo & $\begin{array}{l}\text { Diagnóstico } \\
\text { preoperatorio }\end{array}$ & Intervención & $\begin{array}{c}\text { Sesión } \\
\text { quirúrgica }\end{array}$ & $\begin{array}{c}\text { Sustancias } \\
\text { intraoculares } \\
\text { utilizadas }\end{array}$ & $\begin{array}{l}\text { Utilización de } \\
\text { material } \\
\text { reesterilizado } \\
\text { con gas plasma }\end{array}$ & Complicaciones \\
\hline 1 & 66 & Varón & $\begin{array}{l}\text { Subluxación } \\
\text { de LIO }\end{array}$ & $\begin{array}{l}\text { Vitrectomía y } \\
\text { sutura a iris } \\
\text { de LIO }\end{array}$ & 1 & BSS, cefuroxima & Sí & $\begin{array}{c}\text { Desprendimiento } \\
\text { de retina }\end{array}$ \\
\hline 2 & 56 & Varón & $\begin{array}{l}\text { Hemorragia } \\
\text { vítrea } \\
\text { y catarata }\end{array}$ & $\begin{array}{l}\text { Vitrectomía, } \\
\text { facoemulsificación } \\
\text { e implante de LIO }\end{array}$ & 1 & $\begin{array}{l}\text { BSS, viscoelástico, } \\
\text { adrenalina, } \\
\text { cefuroxima, } \\
\text { bevacizumab }\end{array}$ & Sí & No \\
\hline 3 & 75 & Mujer & $\begin{array}{l}\text { Membrana } \\
\text { epirretiniana } \\
\text { y catarata }\end{array}$ & $\begin{array}{l}\text { Vitrectomía, } \\
\text { facoemulsificación } \\
\text { e implante de LIO }\end{array}$ & 2 & $\begin{array}{l}\text { BSS, viscoelástico, } \\
\text { adrenalina, cefuroxima, } \\
\text { azul tripán }\end{array}$ & 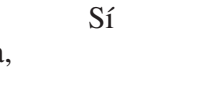 & No \\
\hline 4 & 80 & Mujer & $\begin{array}{l}\text { Hemorragia } \\
\text { vítrea }\end{array}$ & Vitrectomía & 2 & BSS & Sí & No \\
\hline 5 & 70 & Varón & $\begin{array}{l}\text { Membrana } \\
\text { epirretiniana }\end{array}$ & Vitrectomía & 2 & BSS, azul tripán & Sí & No \\
\hline
\end{tabular}

LIO: Lente intraocular. BSS: Solución salina balanceada. 


\section{DISCUSIÓN}

La inmensa mayoría de los brotes de STSA descritos hasta la fecha han aparecido después de cirugía no complicada de catarata, aunque también se ha comunicado algún caso después del implante de lentes fáquicas (3).

El brote que presentamos comprende a un grupo heterogéneo de pacientes en tres de los cuales se realizó cirugía del segmento anterior, pero cuyo nexo común era haber sido sometidos a una vitrectomía posterior.

Entre las causas responsables de la aparición del STSA está cualquier sustancia que pueda ser introducida dentro del ojo durante la cirugía (BSS, adrenalina, viscoelásticos, anestésicos o antibióticos). También puede estar producido por problemas relacionados con los sistemas de limpieza y esterilización del instrumental o por residuos de los detergentes o del óxido de etileno $(4,5)$. La utilización de agua del grifo en el baño de ultrasonidos es otra posible etiología. El agua del grifo contiene niveles bajos de bacterias gram-negativas que pueden reproducirse y originar una endotóxina resistente a la esterilización y que en última instancia sería la causa del síndrome.

En nuestro caso los factores comunes en los cinco pacientes y que podrían explicar la aparición del brote eran la utilización de BSS elaborado en la farmacia del hospital y el uso de material reesterilizado mediante gas plasma y previamente limpiado en una cubeta de ultrasonidos con agua del grifo. Tras la aparición del brote, se sustituyó el BSS por otro similar de elaboración comercial y se comenzó a irrigar la cubeta de ultrasonidos con agua estéril. Se realizó un análisis microbiológico del BSS y un análisis de endotoxinas tanto del BSS como del agua del grifo y aunque los resultados no arrojaron luz sobre la etiología, hasta el momento no hemos detectado nuevos casos. Parece lógico considerar como causa de este brote de STSA el BSS o las endotoxinas formadas en el agua del baño de ultrasonidos.

Lo peculiar del brote que describimos es que aunque en tres de los afectados se había realizado cirugía de segmento anterior, el nexo común es su aparición después de cirugía vítrea. No hemos encontrado en la literatura ningún caso publicado después de cirugía vítrea, de ahí la relevancia de esta comunicación. Resulta difícil explicar la aparición de STSA en pacientes sometidos de forma exclusiva a un procedimiento quirúrgico del segmento posterior, pero es importante pensar en esta entidad ante cualquier reacción inflamatoria desproporcionada del segmento anterior, sobre todo si su aparición cursa en brotes. En conclusión, debemos tener en cuenta que la aparición de STSA también es posible después de cirugía vítrea. Considerar esta posibilidad diagnóstica en lugar de una endoftalmitis infecciosa, de la cual puede ser difícil de distinguir, nos puede evitar tratamientos más agresivos.

\section{BIBLIOGRAFÍA}

1. Mamalis N, Edelhauser HF, Dawson DG, Chef J, LeBoyer $R M$, Werner L. Toxic anterior segment syndrome. J Cataract Refract Surg 2006; 32: 324-333.

2. Holland SP, Morck DW, Lee TL. Update on toxic anterior segment syndrome. Curr Opin Ophthalmol 2007; 18: 4-8.

3. Moshirfar M, Whitehead G, Beutler BC, Mamalis N. Toxic anterior segment syndrome after Verisyse iris-supported intraocular lens implantation. J Cataract Refract Surg 2006; 32: 1233-1237.

4. Hellinger WC, Hassan SA, Bacalis LP, Thornblom DM, Beckmann SC, Blackmore C, et al. Outbreak of toxic anterior segment syndrome following cataract surgery associated with impurities in autoclave steam moisture. Infect Control Hosp Epidemiol 2006; 27: 294-298.

5. Ünal M, Yücel I, Akar Y, Öner A, Altin M. Outbreak of toxic anterior segment syndrome associated with glutaraldehyde after cataract surgery. J Cataract Refract Surg 2006; 32: 1696-1701. 
